\title{
Affinità elettive. Sulla ricezione delle opere di Bassani nei paesi di lingua tedesca
}

Wahlverwandtschaften. Zur deutschsprachigen Rezeption der Werke Giorgio Bassanis

Affinités électives. Sur la réception de l'œuvre de Bassani dans les pays d'expression allemande

\section{Olaf Müller}

\section{OpenEdition \\ Journals}

Edizione digitale

URL: http://journals.openedition.org/cei/3817

DOI: $10.4000 /$ cei.3817

ISSN: 2260-779X

Editore

UGA Éditions/Université Grenoble Alpes

\section{Edizione cartacea}

ISBN: 978-2-37747-040-2

ISSN: 1770-9571

Notizia bibliografica digitale

Olaf Müller, «Affinità elettive. Sulla ricezione delle opere di Bassani nei paesi di lingua tedesca», Cahiers d'études italiennes [Online], 26 | 2018, online dal 28 février 2018, consultato il 27 mars 2021. URL: http://journals.openedition.org/cei/3817 ; DOI: https://doi.org/10.4000/cei.3817

Questo documento è stato generato automaticamente il 27 mars 2021

(c) ELLUG 


\section{Affinità elettive. Sulla ricezione delle opere di Bassani nei paesi di lingua tedesca ${ }^{1}$}

Wahlverwandtschaften. Zur deutschsprachigen Rezeption der Werke Giorgio

Bassanis

Affinités électives. Sur la réception de l'œuvre de Bassani dans les pays

d'expression allemande

\section{Olaf Müller}

1 Giorgio Bassani continua a essere uno degli autori italiani della seconda metà del XX secolo di maggior successo sul mercato librario tedesco. Eppure, ancora oggi, non esiste uno studio sulla ricezione della sua opera in area germanofona. La bibliografia di Portia Prebys ${ }^{2}$, che segnala più di 400 titoli in tedesco fra il 1960 e il 2010, mostra in maniera inequivocabile come l'interesse della critica per l'opera di Bassani sia gradualmente scemato a partire dal 1975. Nonostante i suoi libri vengano ripubblicati con regolarità ancora oggi, e benché questa Bibliografia, talvolta stilata in maniera 'generosa', citi spesso più volte lo stesso titolo o inserisca articoli di testate sicuramente minori come il «Landeskriminalblatt» $»^{3}$ o il «Norddeutsche Schlachter-Zeitung» ${ }^{4}$, questa tendenza sembra evidente.

In una recensione del 1978 alla traduzione tedesca della Sconclusione di Giorgio Manganelli ${ }^{5}$, il noto poeta e saggista Michael Krüger (più tardi direttore dello Hanser Verlag di Monaco) offriva una testimonianza importante a questo proposito, all'interno di un breve sguardo panoramico su una serie di autori italiani contemporanei che erano stati 'dimenticati' dopo aver ottenuto un grande successo nella Germania del dopoguerra. Il giudizio di Krüger è particolarmente interessante, poiché proviene da un grande conoscitore della letteratura italiana che, più tardi, durante la sua attività presso Hanser Verlag, si impegnerà attivamente per dare un ruolo preminente a questa letteratura nel catalogo ${ }^{6}$ della casa editrice. Contrariamente a ciò che avveniva in Francia - scrive Krüger - alcuni classici della letteratura italiana del dopoguerra non 
avevano «nessun mercato» in Germania, e fra questi vi era, nel 1978, anche Giorgio Bassani:

Manganelli conosce in Germania la sorte di molti noti autori internazionali, quella di essere letto solo da specialisti e colleghi. La letteratura italiana - da Cesare Pavese a Giorgio Bassani, da Paolo Volponi a Leonardo Sciascia, da Ungaretti a Montale - era fino a pochi anni fa una lettura obbligata per l'intellighenzia tedesca; ma ora, nonostante i meriti che le sono ininterrottamente riconosciuti, per esempio in Francia, non sembra avere in Germania un mercato abbastanza importante per essere sostenuta dalle istituzioni culturali ${ }^{7}$.

Vedremo che il «valore di mercato» di Bassani era destinato a aumentare nuovamente alcuni anni dopo, in seguito alla trasposizione cinematografica de Gli occhiali d'oro, ma la diagnosi di Krüger è sostanzialmente esatta.

In questo contributo cercheremo in un primo tempo di presentare brevemente, anche sul piano quantitativo, le diverse fasi della ricezione tedesca di Bassani; in un secondo momento, ci concentreremo sui nuclei contenutistici privilegiati dalla critica tedesca; infine, analizzeremo i pochi casi di ricezione letteraria 'creativa' dell'opera di Bassani fra gli autori di lingua tedesca.

\section{Una ricezione quantitativamente importante}

Della prima edizione tedesca de Gli occhiali d'oro, che uscì nel 1960 con il titolo Ein Arzt aus Ferrara ${ }^{8}$, nella traduzione di Herbert Schlüter, si contano fino al 1962 più di 60 recensioni; della traduzione tedesca de Il giardino dei Finzi-Contini e delle Storie ferraresi, che furono pubblicati rispettivamente alla fine del 1963 e del $1964^{9}$, se ne contano circa 40 fino al 1964. Nel 1965 vide la luce un elegante album fotografico, che contiene testi scritti da Bassani e dal suo amico Mario Soldati per le fotografie di Gianni Berengo Gardin ${ }^{10}$. Sempre nello stesso anno, Deutscher Bücherbund di Stoccarda e Buchklub Ex libris di Zurigo ottennero la licenza per una nuova edizione del Giardino ${ }^{11}$, a conferma del grande successo del romanzo sul mercato librario tedesco. Nonostante questo interesse, nella stampa del 1965 il nome di Bassani figura solo in 5 articoli, mentre addirittura non compare in quella del 1966. Nel 1967 e 1968 troviamo di nuovo circa 40 interventi su Bassani, più o meno lunghi, i quali sono in gran parte reazioni alla traduzione di Dietro la porta uscita presso Piper Verlag ${ }^{12}$ e curata, come le precedenti, da Herbert Schlüter. Nel 1967, inoltre, Fischer-Verlag di Francoforte pubblicò Die Gärten der Finzi-Contini in un'edizione tascabile ${ }^{13}$ : di fatto era questa la quarta ristampa del romanzo in cinque anni.

Il 1969 fu un anno particolarmente importante poiché Bassani ricevette il premio NellySachs dalla città di Dortmund. L'onorificenza era stata istituita nel 1961 ed era già molto nota, ma divenne ancor più prestigiosa quando Nelly Sachs ricevette il premio Nobel per la letteratura nel 1966. La Laudatio fu tenuta da Alfred Andersch (1914-1980), uno degli autori tedeschi più noti del dopoguerra, che dal 1934 era stato varie volte in Italia, fra l'altro come soldato della Wehrmacht nel $1944^{14}$, e quindi per un lungo soggiorno romano fra il 1962 e il $1963^{15}$. Andersch, che a sua volta aveva ricevuto il premio Nelly-Sachs nel 1967, svolse un ruolo fondamentale nella nomina di Bassani, da lui conosciuto personalmente durante il periodo trascorso a Roma ${ }^{16}$. Il premio, assegnato nel dicembre del 1969, insieme all'edizione tedesca de L'Airone [Der Reiher] nel 1970, sempre nella traduzione di Herbert Schlüter, segnò il culmine dell'interesse per l'opera di Bassani in Germania, con circa 80 tra recensioni e articoli. 
Particolarmente interessante fu, nello stesso periodo - siamo nel 1970 - l'uscita, in un'edizione su licenza, dei Finzi-Contini presso la casa editrice Volk und Welt nella DDR, seguita nel 1973, sempre su licenza, delle Storie Ferraresi [Ferrareser Geschichten], ambedue con una postfazione di Joachim Meinert, lettore e specialista per la lingua italiana presso questa casa editrice ${ }^{17}$. Queste postfazioni, sulle quali tornereremo in seguito, avevano l'obiettivo di presentare l'opera di Bassani ai lettori della DDR non come un esempio di letteratura borghese e decadente, ma come una lettura ideologicamente edificante.

6 In seguito alla pubblicazione, nel 1974, de L'odore del fieno ${ }^{18}$, uscirono fino al 1975 circa 40 articoli, anche se non tutti incentrati sulla figura di Bassani, mentre a partire dal 1976, e per i cinque anni seguenti, regnò il silenzio sulla sua opera, come confermato appunto da Michael Krüger nel $1978^{19}$. Solo con la seconda ristampa dei Finzi-Contini nella DDR ${ }^{20}$ tornò sul mercato librario un titolo di Bassani - senza che a questo facessero seguito, tuttavia, particolari riscontri nella critica.

7 Fra gli anni 1982 e 1985 registriamo un solo articolo su Bassani, ma non destinato al grande pubblico, perché ospitato in una rivista accademica di italianistica ${ }^{21}$. Anche le nuove edizioni dei Gärten der Finzi-Contini e delle Ferrareser Geschichten ${ }^{22}$ (rispettivamente 1984 e 1985) non suscitarono alcuna reazione. A riprova di questo declino della fortuna di Bassani in Germania, osserviamo che né la nuova stesura delle Storie ferraresi, uscita in Italia con il titolo Dentro le mura nel 1973, né la risistemazione in un solo volume di tutti i suoi romanzi e racconti ferraresi, pubblicati con il titolo Il romanzo di Ferrara nel 1974 e, nella loro veste definitiva nel 1980, furono mai tradotte in Germania. Ancora oggi si leggono le traduzioni delle prime versioni.

Nel 1986 un viaggio di Bassani a Colonia lasciò solo alcune tracce nella stampa locale, ma con la trasposizione cinematografica de Gli occhiali d'oro nel 1987 la presenza dello scrittore nella stampa tedesca si fece più significativa. Il film proponeva fra $\mathrm{i}$ protagonisti volti popolari nella Germania degli anni Ottanta, come Philippe Noiret e Rupert Everett; di conseguenza, per un certo tempo, anche l'autore del romanzo su cui si basava il film poté godere di un'accresciuta attenzione mediatica. Questo rinnovato interesse spinse nel 1991 Piper Verlag di Monaco a ripubblicare le opere di Bassani già uscite, unitamente a un'edizione bilingue di una scelta delle sue poesie ${ }^{23}$; nello stesso anno, il politologo e specialista di Bassani Eberhard Schmidt diede alle stampe una raccolta di saggi critici sull'autore ferrarese insieme a testi autobiografici dello stesso Bassani $^{24}$. Questa timida riscoperta produsse, nel 1991, una nuova serie di recensioni (Portia Prebys ne conta 16). Negli anni successivi solo l'ottantesimo compleanno di Bassani nel 1996, che coincideva con il novantesimo del suo traduttore tedesco Herbert Schlüter, e la vertenza giuridica che contrappose negli anni 1998-1999 i figli di Bassani e Portia Prebys interessò, seppur marginalmente, la stampa tedesca.

Nel 2000, anno della morte, 35 articoli marcarono l'ultima grande ondata d'interesse per Bassani in Germania, e le sue opere trovarono una nuova casa editrice. Piper Verlag, che curava l'opera di Bassani dal 1960, era stato comprato nel 1995 dal gruppo editoriale svedese Bonnier e aveva ceduto i diritti delle traduzioni di Bassani alla casa editrice di Berlino Wagenbach, che dal 2000 si impegna attivamente per mantenere viva la presenza dell'autore sul mercato tedesco ${ }^{25}$. Non a caso Klaus Wagenbach, nato nel 1930 e direttore dell'omonima casa editrice fino al 2002, è un italofilo e un fine conoscitore della letteratura italiana, molto noto in Germania. La sua casa editrice, fondata nel 1964, è diventata negli anni settanta la più importante mediatrice della 
cultura e della letteratura italiana in Germania, e propone molti titoli nel proprio catalogo. Piper pubblicava, oltre a Bassani, altri autori importanti quali Ungaretti, Gadda, Quasimodo, Montale o Tomasi di Lampedusa, ma erano nomi isolati all'interno di un programma editoriale non incentrato sull'Italia ${ }^{26}$. Presso Wagenbach, invece, l'opera di Bassani divenne uno dei pilastri di un catalogo già molto orientato verso la letteratura italiana. L'importanza dello scrittore ferrarese per questo editore traspare ancor oggi dalla frequenza con cui i suoi titoli vengono riproposti: Die Gärten der FinziContini è uscito per Wagenbach nel 2000, poi nel 2008 e ancora nel $2016^{27}$, Die Brille mit dem Goldrand nel 2007 e nel 2016, con un'immagine tratta dal film con Philippe Noiret in copertina. Nel 2007 è stato ripubblicato anche L'Airone presso la stessa casa editrice ${ }^{28}$, cui è seguita nel 2008 l'edizione di Dietro la porta ${ }^{29}$. Nel 2009, infine, ha visto la luce, sempre presso Wagenbach, un'antologia curata da Carl Wilhelm Macke, che include anche un testo di Bassani ${ }^{30}$.

Nel 2016, in occasione del centenario dalla nascita, l'autore è stato ricordato sia attraverso una serie di articoli sulla stampa generalista, sia attraverso mostre e conferenze curate dagli Istituti di cultura italiana di Monaco e Berlino. Nonostante queste manifestazioni e una continua richiesta dell'opera bassaniana da parte del pubblico di lingua tedesca, che trova riscontro nella ristampa delle sue opere ${ }^{31}$, i media e, per certi versi, anche la critica letteraria, si mostrano da qualche anno poco ricettivi ${ }^{32}$.

\section{I temi principali della ricezione in lingua tedesca}

11 Quali sono i temi principali delle risposte in lingua tedesca all'opera di Bassani a partire dal 1960? La prima recensione in assoluto si riferisce alla traduzione tedesca de Gli occhiali d'oro, e apparve in una pubblicazione non specificamente letteraria ma legata a uno dei principali temi del romanzo: si tratta di una rivista destinata a un pubblico omosessuale, «Der Kreis», fondata nel 1932 a Zurigo. L'analisi del romanzo di Bassani, che apparve nel numero del dicembre 1960, si concentrava prevalentemente sulla rappresentazione dell'omosessualità. Di solito gli autori degli articoli della rivista pubblicavano sotto pseudonimo ${ }^{33}$ : il recensore de Gli occhiali d'oro, invece, firmò col suo vero nome, Christian Helder, e consigliò il libro sia ai lettori della rivista sia a tutti i sostenitori del movimento, vedendo nel protagonista del romanzo, il dott. Fadigati, un modello per tutti gli omosessuali [«Schwule»] vittime di esclusione sociale:

Questo racconto è una lettura che raccomandiamo ai nostri lettori. Si può dare il libro addirittura ai 'nemici', perché ci mette in buona luce. Questo lavoro di Bassani risulta particolarmente gradito perché, per una volta, la persona rappresentata non ha tratti decadenti, come in molti altri autori. Il 'medico di Ferrara' è rispettato e compreso dalla sua comunità, anche quando si sa che lui è 'così'. Dovrebbe diventare un modello per noi, perché ci mostra come, in mezzo ai comportamenti ostili, si possa preservare la forza del carattere e agire nobilmente ${ }^{34}$.

Incredibilmente, la discriminazione antisemita a cui è esposto il narratore, alla vigilia della promulgazione delle leggi razziali nel 1938, in evidente parallelismo con la persecuzione omofoba di Fadigati, non sembra avere alcuna importanza per il recensore. Chi leggesse la recensione senza conoscere il romanzo, non capirebbe che il narratore è ebreo, e potrebbe esserne indotto a pensare che in Italia anche i cattolici siano stati perseguitati sotto il fascismo. Helder dice soltanto, molto vagamente, che Bassani 
[...] vorrebbe difendere la causa dei perseguitati e dei proscritti, ma non vuole che la sua persona in quanto autore sia troppo associata a loro. Cerca di spiegare molti conflitti attraverso una rappresentazione precisa del contesto politico - l'atteggiamento dei fascisti italiani nei confronti dei cattolici e degli ebrei svolge un ruolo importante nel racconto ${ }^{35}$.

13 Altrettanto fuori luogo è il giudizio di Helder quando alla fine afferma che la rappresentazione della persecuzione della comunità ebraica di Ferrara cela «il nucleo centrale del racconto» ${ }^{36}$, da lui ovviamente identificato con l'omosessualità di Fadigati: alla luce delle sue preoccupazioni specifiche, la tematica dell'antisemitismo e le leggi razziali del 1938 sembrano al recensore una fastidiosa appendice.

Anche l'austriaco Franz Tumler, che era stato un fedele, e molto amato, sostenitore del regime nazista, ha nella sua recensione del 1961 difficoltà a riassumere il contenuto de Gli occhiali d'oro. Il suo giudizio sul romanzo è nel complesso molto positivo, ma si concentra esclusivamente sul problema dell'isolamento [«Vereinsamung»], come indica il titolo dell'articolo. Fadigati e il narratore sono per Tumler degli individui marginalizzati dalla società, ma il recensore non riesce a designare apertamente l'omosessualità del protagonista, e parla molto vagamente delle «sue innate biasimevoli tendenze» [«die ihm angeborenen verpönten Neigungen»] ${ }^{37}$. Certo, l'isolamento del narratore è ricollegato alle sue origini ebraiche, e sono queste stesse «origini ebraiche, che attraverso la pressione delle persecuzioni allora decretate in Italia, lo isolano improvvisamente dalla società in cui era cresciuto», per cui Bassani mostra «il silenzioso progresso della fatalità [«Verhängnis»], mentre esteriormente tutto sembra immutato ${ }^{38}$. Ma il tema dell'antisemitismo non è mai direttamente affrontato. La riflessione rimane così generica che il lettore non viene informato né del fatto che Bassani aveva condiviso anche personalmente le sofferenze degli ebrei di Ferrara, né che la legislazione antisemitica in Italia era stata adottata di concerto con la Germania, cosa che invece nel testo di Bassani è esplicitamente indicata.

Per i primi recensori tedeschi, Bassani è stato innazitutto lo scopritore del Gattopardo, che uscì in Germania nel 195939; anche l'edizione tedesca dei racconti di Tomasi di Lampedusa, pubblicati nel 1963, aveva una postfazione di Bassani: un elemento che rafforzò nel pubblico il legame tra questi due autori ${ }^{40}$.

16 Ci si potrebbe dunque legittimamente aspettare che il nome di Bassani fosse abbastanza diffuso in Germania, in particolare fra i letterati attivi intorno al 1960, poiché nella sua funzione di redattore della rivista «Botteghe oscure», egli fu coinvolto, fra il 1948 e il 1960, nella pubblicazione di importanti autori di lingua tedesca: Ilse Aichinger, Ingeborg Bachmann, Max Bense, Horst Bienek, Heinrich Böll, Paul Celan, Hilde Domin, Hans Magnus Enzensberger, Günter Grass, Helmut Heißenbüttel, Walter Höllerer, Uwe Johnson, Marie Luise Kaschnitz, Karl Krolow, Christoph Meckel, Heinz Piontek, Nelly Sachs fino a Martin Walser e Franz Wurm, solo per citare i più importanti ${ }^{41}$; a ben vedere, però, solo pochi tra questi autori ebbero contatti diretti con Bassani - almeno a giudicare dal materiale finora pubblicato. Ingeborg Bachmann e Paul Celan, che pubblicarono poesie su «Botteghe oscure» a partire rispettivamente dal $1954^{42} \mathrm{e}$ dal 1956, curarono addirittura, nel 1958, un numero monografico della rivista sulla lirica tedesca contemporanea. Tuttavia, il nome di Bassani non figura nella loro corrispondenza.

17 Nel 1962 un altro dibattito letterario attira l'attenzione del pubblico tedesco sul nome di Bassani, ponendolo però in una luce non del tutto positiva: circolò, infatti, la notizia 
che Bassani si fosse dato da fare perché il romanzo di Anna Banti Le mosche d'oro ottenesse il Premio Strega, mentre lui stesso era in lizza per il Premio Viareggio. L'articolo ricordava i titoli di Bassani e la scoperta del Gattopardo, ma proponeva anche un quadro poco edificante di attori influenti della scena letteraria che intrigano per assicurarsi i premi letterari del 1962 e favorire i propri conoscenti:

Giorgio Bassani, autore di raffinati testi letterari [«literarisch geschliffener Bücher»] (Geschichten aus Ferrara, Der Garten der Finzi-Contini), editore di una delle più importanti collane editoriali, quella dei «Narratori moderni» presso Feltrinelli, e rispettato da molti come esperto infallibile di cose letterarie in seguito al lancio del Gattopardo, che era stato rifiutato più volte da altri editori, corre il pericolo di veder intaccata la sua reputazione. Le sue proposte sono di parte, legate a fini personali. Dieci giorni prima dell'assegnazione del premio Strega si è impegnato alacremente per promuovere Le Mosche d'oro [Die Goldfliegen] di Anna Banti, che fino a poco tempo prima si rifiutava di leggere. Il libro ha avuto solo il terzo posto. Circola voce che Anna Banti troverà il modo di vendicarsi, anche di Bassani, che con tutta l'energia della sua ambizione spera di aggiudicarsi il Premio Viareggio (dotato di quattro milioni di lire) per il suo Il Giardino dei Finzi-Contini ${ }^{43}$.

Questo pettegolezzo sugli intrighi letterari italiani non fu però di ostacolo alla diffusione del libro in Germania, che uscì in traduzione un anno dopo. La ricezione del romanzo fu unanime. Un breve articolo dello «Spiegel» del 25 dicembre 1963, che ricordava Bassani come lo scopritore del Gattopardo, definiva tuttavia il suo stile narrativo «kunstvoll konventionell» [«artificiosamente convenzionale»], espressione ambivalente quanto i «raffinati testi letterari» dell'articolo di Monika von Zitzewitz sui premi letterari. Il romanzo, proseguiva l'articolo, racconta la

[...] storia di una famiglia ebrea di Ferrara, che, all'inizio dell'era fascista, fa dei suoi famosi giardini, e più tardi anche della propria biblioteca, il luogo d'incontro di onorati cittadini, fra i quali si sviluppa a poco a poco un'atmosfera di tacita resistenza passiva. Un'infelice storia d'amore fra la figlia dei proprietari e il narratore domina il romanzo dalle tonalità autobiografiche. I giardini dei FinziContini cadono nell'abbandono quando la famiglia viene deportata in Germania ${ }^{44}$.

Il fatto che il singolare del titolo italiano sia trasformato, nella traduzione tedesca, nel plurale Giardini [Gärten] non sembra disturbare il recensore, benché ciò faccia perdere uno degli strati semantici più importanti del giardino dei Finzi-Contini, e cioè il suo carattere paradisiaco. È interessante constatare che questo errore non è mai stato corretto. Ma almeno la recensione menziona la deportazione dei Finzi-Contini in Germania, pur tacendo la loro uccisione. Sempre su «Die Zeit», nella rubrica «La voce del libraio», alla fine del 1963, il romanzo di Bassani fu consigliato da molti librai tedeschi, senza mai fare riferimento alla persecuzione degli ebrei italiani da parte del regime fascista, a partire dal 1938, e alla successiva uccisione delle famiglia citata nel titolo. Alla domanda «quali libri consiglierà ai suoi clienti per l'autunno 1963», così rispose una libraia di Düsseldorf: «Consiglierò Giorgio Bassani: "Die Gärten der FinziContini" (Piper Verlag). Perché? Per la lingua poetica e la bellissima rappresentazione del mondo, lacerato da segrete fratture, di una colta famiglia ebrea di Ferrara nel $1935-36 »$.

L'errata collocazione cronologica degli eventi segnala già, in questa dichiarazione, che la problematica centrale del romanzo non è stata colta, come mostra anche l'assurdo eufemismo «mondo lacerato da segrete fratture» che potrebbe indicare dei contrasti familiari o altre simili banalità. Altrettanto vago si mostrò un libraio di Amburgo, al 
quale forse sembrò inopportuno fare menzione del fascismo e della persecuzione degli ebrei fra i temi del romanzo:

La bellezza e la grazia di questi ricordi di una vita in una famiglia ebrea di Ferrara nascono da una particolare tristezza, con la quale l'autore ripercorre il passato e l'impossibilità del ritorno della felicità perduta. Bassani è riuscito a riunire in questo romanzo prosa e poesia ${ }^{46}$.

Una recensione molto accurata uscì, invece, nel 1962 su «Tribüne. Zeitschrift zum Verständnis des Judentums». La rivista era stata fondata per contrastare con la documentazione e l'analisi l'antisemitismo sempre presente e le ringagliardite tendenze neonaziste della Germania degli anni Sessanta. Il redattore Axel Silenius scrisse in un intervento dal titolo Die Erinnerung der "verlorenen Zeit» [Il ricordo «del tempo perduto»] che la memoria era «il centro del romanzo», e stava lì per compensare «la tomba, la lapide di coloro che, nella distruzione e nella rovina, non avevano neppure potuto trovare, come estremo rifugio, "una sepoltura qualsiasi" ${ }^{47}$.

Una recensione non meno approfondita fu scritta da Wolfgang Grözinger nel dicembre 1963 per la rivista cattolica «Hochland». L'autore conosceva Bassani come scopritore del Gattopardo, oltre che come poeta e redattore di «Botteghe oscure» e «Paragone». Nell'articolo sono chiaramente indicati i legami fra il fascismo e l'antisemitismo italiani e tedeschi, sfondo indispensabile alla comprensione della vicenda:

Il poeta e critico italiano Giorgio Bassani, redattore di due riviste letterarie e scopritore di Der Leopard di Lampedusa, ha ottenuto un grande successo in patria e all'estero con il suo primo romanzo Die Gärten der Finzi-Contini. Questo successo è meritato. Quando uno prende il libro in mano, ha l'impressione di incontrare quella forma di libertà che si chiama nobiltà interiore. È sconvolgente che questa disposizione di spirito si manifesti in tutta la sua forza sul margine dell'abisso nel quale l'antisemitismo hitleriano ha precipitato anche gli ebrei italiani ${ }^{48}$.

Grözinger identifica con grande precisione il terribile contesto entro cui si doveva leggere la storia raccontata da Bassani, storia solo apparentemente banale, e descrive in modo pertinente la tecnica dell'evocazione utilizzata dallo scrittore. Il confronto conclusivo con lo stile del Gattopardo può sembrare forzato, ma se si osserva il tono melancolico di entrambi i testi, non è troppo lontano dalla verità:

\footnotetext{
Qui, una volta tanto, non ci viene mostrata la vittima meschina e impaurita dell'odio razziale, che dal ghetto e dal pogrom arriva direttamente nell'inferno di Auschwitz, bensì la nobiltà ebraica. [...] È commovente constatare come Bassani sia riuscito a tal punto a smorzare lo strepito della politica di quegli anni attraverso il motivo della disubbedienza aristocratica che la voce sottile di un infelice amore giovanile, il tema eterno del giovane che vuole afferrare le stelle diventa qui udibile. Bassani ha scritto in questo modo - e forse senza il modello di Lampedusa questo non sarebbe stato possibile - il pendant ebraico del Leopard, un libro che, per quanto paradossale ciò possa sembrare, rallegra attraverso la rinuncia e il dolore $^{49}$.
}

24 A fine 1964 l'edizione tedesca delle Storie ferraresi veniva annunciata in una breve recensione dello «Spiegel» come l'opera dello «scopritore di Lampedusa (Der Leopard) Bassani ${ }^{50}$. Il pubblico riceveva però, da questa recensione, solo informazioni molto vaghe e di scarso interesse, come quella che nelle Storie Ferraresi «il passato della provincia italiana» veniva «superato» e che

Gli eroi passivi di Bassani, [...] ebrei o fascisti, sono comunque degli outsider. Tutti soffrono di inibizioni sessuali [erotische Befangenheit]. Le donne si muovono fra chiesa e cucina. Gli uomini hanno paura della vita. Bassani dà il meglio di sé 
quando, in uno stile un po' laborioso, riesce a rendere poetica l'oscura e malinconica atmosfera di Ferrara ${ }^{51}$. dei Finzi-Contini presso Fischer Verlag di Francoforte ${ }^{53}$, reagirono nel 1967-1968 circa 40 recensioni, ma il successo tedesco più importante per Bassani in quegli anni fu senza dubbio il conferimento del premio Nelly-Sachs della città di Dortmund, che l'autore ritirò nel dicembre 1969. La Laudatio fu fatta da Alfred Andersch, che conosceva Bassani da molti anni ${ }^{54}$ e che parlò di «una certa affinità elettiva» con l'autore ferrarese. Come ha sottolineato Italo Michele Battafarano, il discorso di Andersch su Bassani «si può leggere anche come una riflessione di Andersch sul suo proprio lavoro e sulla sua concezione della letteratura $\aleph^{55}$, in quanto Bassani diventa per l'autore tedesco una sorta di «alter ego italiano» ${ }^{56}$. La Laudatio di Andersch fa tuttavia capire sin dall'inizio che Bassani, nel 1969, non era ancora un autore pienamente affermato presso il pubblico tedesco. Già nella prima frase, dopo avere precisato che non farà «un discorso ufficiale», afferma Andersch:

Non ho altro fine che raccontarvi di Giorgio Bassani e Ferrara [...] e vi lascerò decidere poi se il conferimento del premio, che va sotto il nome della nostra grande e indimenticabile Nelly Sachs, è giustificato o se si tratta di un'idea strampalata [«skurrilen Einfall»] ${ }^{57}$.

Se perfino il laudator ammette che per il pubblico del 1969 il conferimento del premio a Bassani possa essere uno «skurrile[r] Einfall», ci si può chiedere quali aspettative egli attribuisse al pubblico e per quale motivo Bassani non sarebbe stato all'altezza. Prima di Bassani avevano ricevuto il premio (che veniva dato ogni due anni) la stessa Nelly Sachs (1961), Johanna Moosdorf (1963), Max Tau (1965) e Alfred Andersch (1967). Tutti e quattro o erano stati vittime dirette della persecuzione antisemita in Germania (Sachs, Moosdorf, il cui marito, il politologo Paul Bernstein, era stato assassinato nel 1944 a Auschwitz, e Tau) oppure (nel caso di Andersch) si erano confrontati intensamente con i crimini tedeschi dopo il 1945. Da questa prospettiva non era affatto «bizzarro» che Bassani ricevesse il premio, ma a quanto pare Andersch non riteneva Bassani abbastanza noto al pubblico perché sembrasse scontato il conferimento del premio. Andersch stesso aveva contribuito due anni prima a far conoscere l'opera di Bassani in Germania: nel «Merkur», un mensile fondato nel 1947 e ancora molto importante nel 1967, egli aveva pubblicato un articolo dal titolo Auf den Spuren der Finzi-Contini ${ }^{58}$, in cui raccontava di una visita a Ferrara durante la quale aveva cercato i luoghi dei racconti di Bassani. La Laudatio del 1969 era una rielaborazione (a volte più breve, ma a volte anche più sostanziosa) di quell'articolo. Era stato Andersch a proporre Bassani per questo premio: nel 1968 aveva avuto uno scambio epistolare con Nelly Sachs a questo proposito e le aveva chiesto di fare il nome di alcuni possibili candidati. Sachs, che aveva ricevuto il premio Nobel due anni prima, scrisse ad Andersch il 7 maggio 1968 e nominò vari autori tedeschi fra cui Paul Celan, Heinrich Böll, Helmut Heißenbüttel, Elisabeth Borchers, Hubert Fichte, Jürgen Becker e Gisela Elsner. Nominò anche Samuel Beckett, che lei stimava molto e considerava come un «fratello», ma che giudicava poco adatto al riconoscimento di Dortmund in quanto non era autore di lingua tedesca ${ }^{59}$.

Cahiers d'études italiennes, 26 | 2018 
Possiamo quindi supporre che non sia stata Nelly Sachs a fare il nome di Bassani, anche se lo poteva conoscere come redattore di «Botteghe oscure», giornale in cui anche lei era stata pubblicata. Forse la frase iniziale sull'«idea strampalata» di conferire il premio a Bassani si riferiva proprio al fatto, ben noto a Andersch, che a Nelly Sachs non sarebbe mai venuto in mente di ricompensare l'autore ferrarese.

Fra la lettera di Nelly Sachs a Andersch del maggio '68 e la cerimonia di consegna del premio nel dicembre 1969 ci fu un incontro molto importante che Bassani stesso ricorda nella sua risposta alla Laudatio di Andersch: il nome di Nelly Sachs gli era noto almeno dal 1959, quando alcune sue poesie erano state pubblicate su «Botteghe oscure»: «Era la prima volta che nel mio paese si stampava qualcosa di suo, dieci anni prima della pubblicazione in volume di sue opere presso Einaudi» ${ }^{60}$. L'incontro fra i due era avvenuto in un ospedale svedese nell'ottobre 1969, poco prima della consegna del premio, e fu la conferma, per l'autore ferrarese, di un suo accordo fondamentale con la poetica di Nelly Sachs:

[...] fu una visita che non dimenticherò, credo, mai, la poetessa era (ed è ancor oggi) molto malata, era quasi diventata un nulla, un minuscolo involucro senza peso. E tuttavia non potrò dimenticare mai i suoi occhi ardenti, vivacissimi, indomiti, il suo volto gracile, pallido, emaciato, ma miracolosamente illuminato dalla luce interiore dello spirito. Non potei rimanere più di qualche istante: ero in piedi accanto al suo letto, e lei mi guardò e mi parlò dal fondo dei suoi cuscini. Appena cinque minuti dopo avere varcato la soglia della sua cameretta, già scendevo insieme al mio amico le scale della clinica. E lì compresi che la poesia è sempre il risultato di privazione [«Entbehrung»], separazione ed esilio. È stato proprio nell'esilio svedese che Nelly Sachs ha trovato la sua Germania. Dall'ospitale Svezia invocava [«beschwor sie»] i morti tedeschi di Buchenwald, Mauthausen, Dachau: le lunghe, verticali, 'espressionistiche' nubi di fumo dei suoi e dei nostri morti. Evocava [«evozierte»] i morti. Ma non è questo - mi dissi allora e mi dico ancor oggi - il destino o meglio, la prima funzione del poeta? Non hanno i poeti, sin da Omero, cantato i morti, nel tentativo disperato e però vincente di ridar loro la vita $^{61}$ ?

Bassani ringraziò ovviamente anche il laudator Andersch e accennò al fatto che buona parte del suo intervento aveva come fonte l'articolo del $1967^{62}$. Verena Kammandel, che ha cercato tracce dell'opera di Bassani negli scritti di Andersch, nota il profilarsi, fra il 1957 (anno del romanzo Sansibar oder der letzte Grund) e il 1967 (anno del romanzo Efraim e del saggio sul Merkur) di «un modo molto più sottile di trattare il tema del passato nazionalsocialista in Germania [...] che deve molto a Bassani» ${ }^{63}$. Anche se non si riscontrano tracce concrete di una ricezione bassaniana nell'opera letteraria di Andersch, sembra legittimo sostenere che l'intenso confrontarsi con la questione della trasmissione della memoria, individuale e «collettiva», evidente anche nella Laudatio, risalga alla scoperta dell'opera di Bassani durante il soggiorno romano dell'autore alla fine del 1962. Così si esprime Andersch nella Laudatio:

Il compito della letteratura - parlo qui della prosa - è dare una forma ai ricordi in un processo narrativo. Cos'è successo nei romanzi di Bassani? È semplicemente accaduto che un grandissimo narratore ha ricordato, che egli ha trasformato i suoi ricordi in ricordi collettivi e che la sua memoria è entrata a far parte della memoria del singolo lettore. Ricordi così ricordati che non li dimentichiamo. Troppo poco? Ma supponiamo che non ci fosse più il ricordo di Pino Barilari o di Micòl FinziContini, di Raskolnikow o Enrico il verde [il protagonista di Der grüne Heinrich di Gottfried Keller], di Emma Bovary e Effi Briest, di Josef K. e Stephen Daedalus - con quale sconsideratezza e incoscienza potrebbe allora l'umanità [Menschheit] abbandonarsi a atti disumani, feroci [Unmenschlichkeiten] ${ }^{64}$ ? 
Avendo già altrove paragonato la Ferrara di Bassani alla Combray di Proust o alla Praga di Kafka, Andersch mette senz'altro l'opera di Bassani sullo stesso piano di quella di Dostoevskij, Gottfried Keller, Flaubert, Fontane, Kafka o Joyce. Ciò che le unisce, a suo giudizio, è il coninvolgimento della memoria personale, che Bassani ha eletto a principio costitutivo della maggior parte dei propri testi.

31 Andersch ripubblicò il saggio su Ferrara del 1967 in un'antologia dal titolo Norden Süden rechts und links [Nord sud destra e sinistra], che uscì nel 1972 per i tipi di Diogenes a Zurigo. Fu questa versione del testo che lesse lo scrittore Jean Améry, amico di Andersch, ex-combattente della Resistenza ed ebreo austriaco perseguitato e torturato dai nazisti, che aveva incontrato Primo Levi ad Auschwitz. Fino ad allora Améry conosceva di Bassani solo la riduzione cinematografica del Giardino dei Finzi-Contini, realizzata nel 1970 da Vittorio De Sica. La lettura del saggio di Andersch su Bassani lo entusiasmò:

Ieri ho letto con sincero entusiasmo il Suo testo su Ferrara e sui Finzi-Contini. Non ho letto il libro di Bassani, confesso con vergogna che non so l'italiano. Ma il film di De Sica l'ho visto e ora il Suo articolo, che è un po' come la ricerca di Combray, che a suo tempo avevo intrapreso, mi ha chiarito molte cose e aperto diverse nuove prospettive - in breve: ho letto con entusiasmo, purtroppo solo da non addetto ai lavori ${ }^{65}$.

Nonostante tutto il suo entusiasmo, Améry non aveva capito che, nel momento in cui scriveva la lettera ad Andersch, Il giardino dei Finzi-Contini era già stato tradotto in tedesco da almeno dieci anni, e neppure il film aveva, evidentemente, risvegliato in lui il desiderio di interessarsi a un libro che avrebbe potuto facilmente leggere anche senza sapere l'italiano, se davvero avesse voluto.

Nel frattempo, nel 1970, era uscita anche la traduzione tedesca de L'Airone. Il romanzo fu accolto come il «capolavoro» di Bassani da critici letterari autorevoli come Toni Kienlechner del giornale «Die Zeit»:

Con questo romanzo Giorgio Bassani si è prefisso più di quanto non avesse fatto in tutte le opere precedenti. Per questo motivo non si è limitato alla sua abituale scrittura scarna. Nei racconti precedenti, dalla prosa tranquilla e distesa, si innalzavano momenti di alta liricità, sufficienti a rendere folgoranti i suoi personaggi. Qui, dove è posto il problema della morte, Bassani ha steso sin dall'inizio un ampio telone, per rappresentare in toto l'immenso contrasto tra vita $\mathrm{e}$ morte: per realizzare il suo capolavoro ${ }^{66}$.

Sempre nel 1970 uscì la prima edizione de Il giardino dei Finzi-Contini nella DDR presso le edizioni Volk und Welt di Berlino. Nella sua postfazione ben informata, il traduttore e specialista di letteratura italiana Joachim Meinert identificava l'introduzione delle leggi razziali nel 1938 come la svolta decisiva nella produzione letteraria di Bassani. Nel suo primo libro Una città di pianura, Bassani aveva utilizzato una "prosa d'arte che attribuiva maggior valore alla ricercatezza linguistica e alla perfezione artistica che non a temi di rilevanza sociale» ${ }^{67}$. Il suo mondo protetto venne scosso «quando nel 1938 in Italia i fascisti scatenarono un'ondata di antisemitismo». L'analisi di Meinert seguiva alcuni criteri tipici di una postfazione scritta nella DDR: egli affermava, ad esempio, che dopo la guerra Bassani si era interessato «a soggetti di maggiore importanza, a temi di rilevanza sociale e morale» e che i suoi racconti, nonostante «si limitassero a descrivere uno specifico ambiente locale e uno strato sociale molto ristretto, la borghesia ebraica», proponevano «una visione universale della situazione della borghesia in una città della provincia italiana ${ }^{68}$. Meinert riconosceva a Bassani il merito di non essersi limitato al 
«ruolo di analista psicologico e cronista sociale», di essersi dischiuso alla poesia "rifugio" indocile» ${ }^{69}$. Nel contesto della politica culturale ufficiale della DDR queste osservazioni sono di per sé degne di nota. Ancor più importante è il fatto che Meinert - il quale già nel 1970 conosceva minuziosamente tutte le opere di Bassanirichiamasse l'attenzione su un'affermazione che in anni successivi sarebbe diventata uno dei punti cardinali della ricezione di Bassani da parte di W. G. Sebald. Meinert cita infatti il passo di un'intervista in cui Bassani aveva definito la propria poetica ${ }^{70}$. Il suo obiettivo, aveva detto, era quello di «accostare il più possibile il documento all'intuizione poetica, fino a far prendere a questa la tonalità di quello» ${ }^{71}$. Quasi trent'anni dopo, nel corso delle sue letture bassaniane, Sebald doveva sottolineare a matita, in un volume di suo possesso, proprio queste parole, che trovava citate in un saggio di Eberhard Schmidt ${ }^{72}$.

Meinert interpretava il rapporto tra Micòl Finzi-Contini e il comunista Malnate come l'espressione della tensione tra "la tentazione di fuggire in un passato poetico» e la «volontà di operare attivamente nel presente» e sottolineava come positivo il fatto che «uno scrittore borghese e antifascista riconoscesse ai comunisti la più lucida coscienza politica» ${ }^{73}$. Dopo una dettagliata presentazione delle opere di Bassani pubblicate fino al 1969, incluso L'Airone, che non era ancora stato pubblicato in Germania e che egli considerava poco riuscito, Meinert tentava di inquadrare il significato della Resistenza per autori di estrazione borghese quali Bassani, e giungeva a questa conciliante conclusione:

Anche se a Bassani non può essere riconosciuta una «coscienza storica matura», $\mathrm{i}$ tratti antifascisti e in parte anche antiborghesi delle sue opere dimostrano che il vasto movimento popolare della Resistenza in Italia ha influenzato in modo sostanziale persino la letteratura di origine borghese ${ }^{74}$.

Quanto alla traduzione tedesca de L'odore del fieno, uscita nel 1974 ad opera di Herbert Schlüter presso Piper Verlag di Monaco, anche Rito Sander, nella sua recensione, ricorse alla metafora del maestro, come già aveva fatto Toni Kienlechner a proposito de L'Airone, e paragonò Bassani a Pavese. Dello stile scarno di Bassani lodò il fatto che

[...] lo sforzo artistico di cancellare le tracce dello sforzo creativo nel prodotto artistico [...] ha spolpato le opere di questo autore italiano fino all'osso. Nemmeno una parola superflua, nulla di opulento, di ridondante: parsimonia eloquente, testi in cui la discrezione induce a cassare tenacemente; risparmio realistico di mezzi, come vuole la tradizione contemporanea di questo paese, per esempio in Pavese ${ }^{75}$.

Attraverso una citazione dai Tre apologhi ne L'Odore del fieno, e sottolineando il sintagma «quasi niente», anche Sanders prendeva atto dell'autorevolezza e maestria di Bassani:

Sono quasi cronache questi racconti quieti, misurati, senza nessuna pompa, attualità raccontate come eventi della vita di tutti i giorni. «Accadde parecchi anni fa, prima dell'apertura delle autostrade. Accadde? Beh, per modo di dire, giacché in realtà non accadde quasi niente». Questo «quasi niente» per noi così importante di cui Bassani è maestro ${ }^{76}$.

Questi giudizi del 1974 coincidono con l'apice della popolarità di Bassani in Germania. Da allora in poi uscirono solo riedizioni, più o meno rielaborate, delle opere già pubblicate, e anche le reazioni dei pubblicisti si fecero sempre più rare. La bibliografia di Prebys non registra alcuna recensione in lingua tedesca negli anni che vanno dal 1976 al 1980. Nel 1980 si registra un saggio molto circostanziato, pubblicato in una rivista letteraria svizzera, ma che si legge quasi come un elogio funebre. Georges Amman vi constatava la scomparsa di Bassani dalla scena letteraria contemporanea. 
Dopo un breve riepilogo dei successi di Bassani nei paesi di lingua tedesca fino al premio Nelly Sachs, l'autore si chiede:

Qual è la ragione del lungo silenzio di Bassani negli anni' 70 , silenzio che ha sicuramente contribuito alla scomparsa di questo scrittore squisitamente sensibile dalla scena letteraria contemporanea? [...] Forse la ricerca di nuovo materiale, di nuove forme non si è ancora conclusa o avanza in segreto, lontano dal pubblico; forse attraverso la questione del senso e dell'ufficio della scrittura è giunto alla conclusione di avere già detto ciò che è davvero importante ${ }^{77}$.

Dopo uno sguardo panoramico su tutte le opere di Bassani e dettagliate informazioni sui contenuti dei racconti più lunghi, Amman torna alla questione iniziale: quale può essere stata la ragione del silenzio di Bassani dopo L'Airone?, e giunge alla conclusione che forse Bassani, come Marcel Proust, non si ritiene capace di

[...] rinunciare al proprio punto di vista di letterato ed esteta che mette da parte le questioni urgenti e assillanti del tempo presente. È possibile che l'autore, così avido di ricordi, e innamorato in modo così esclusivo del proprio passato, non sia capace di mettere la propria sensibilità al servizio di una comprensione del mondo che sia più attuale e più risoluta, che si addentri fin nelle viscere delle $\operatorname{cose}^{78}$ ?

Che le ragioni del silenzio di Bassani fossero da ricercare piuttosto nella sua malattia, divenne di pubblico dominio anche nei paesi di lingua tedesca solo negli anni '90. Un articolo di Gustav Seibt sulla «Frankfurter Allgemeine Zeitung» provocò nel 1995 molto scalpore. Seibt era andato a far visita a un Bassani ormai affetto da demenza nella sua abitazione romana e in mezzo all'incessante chiacchiericcio degli altri invitati aveva cercato di avvicinarsi all'autore e sottrarlo per qualche momento al suo ottenebramento mentale. Dopo quello che all'ospite tedesco parve un promettente inizio, Bassani scomparve di nuovo dietro il cicaleccio degli altri invitati:

La serata era iniziata in modo così commovente. Ancora prima dell'arrivo della loquacissima signora inglese, l'anziano signore, non sapendo quali fossero le conoscenze pregresse dei suoi ospiti, era andato a prendere in una stanza attigua l'edizione in un solo volume di tutta la sua opera narrativa, Il Romanzo di Ferrara, che racconta la storia della sua città natale e degli ebrei che vi abitavano sotto il fascismo, e si era affacciato alla porta con le parole: «Ecco il mio poema». Teneva in mano tutta la sua vita, un migliaio di pagine tristi e sconvolgenti, Il giardino dei FinziContini e gli altri racconti, tutta la sua meravigliosa opera. Stava lì come uno degli antichi poeti nel Limbo dantesco. Dopodiché si era accasciato in una poltrona e congedato dal tempo presente. Presto diventa chiaro che gli altri invitati sono lì per celare la sua situazione infelice. Si dimentica le cose all'istante, chiede cinque volte il nome e la provenienza dei suoi ospiti, dà risposte strascicate e indistinte ${ }^{79}$.

41 Il resoconto di Seibt termina con la descrizione di un bacio che Bassani gli aveva dato in un momento in cui i due erano riusciti a sottrarsi alle chiacchiere degli altri invitati. Dopo la pubblicazione del'articolo nel 1995, la comicità involontaria di questo epilogo suscitò reazioni divertite in altri giornali tedeschi, anche se l'asserzione centrale che Bassani trascorreva la maggior parte della sua esistenza in uno stato di ottenebramento mentale era in sé tutt'altro che divertente:

Siamo soli. Ora gli faccio una confessione. Amo tutti i suoi libri, però nessuno mi ha turbato quanto Dietro la porta. Vi ho riconosciuto tutta la mia vita. Bassani mi guarda tranquillo. Si sporge in avanti e dice: «Davvero? Sì, è una storia triste. Ma importante. Molto importante». Ora attira rapidamente, con mia grande sorpresa, il mio capo verso di sé e mi dà un bacio sulla guancia. Anch'io gli do un bacio, sulla guancia sinistra, e vorrei sfiorare anche la destra. Ma lui si ritrae e dice: «No, solo una guancia!» [...] Mi accomiato rapidamente [...]. Bassani mi segue fino all'ingresso, dove prendo il cappotto. Quando sono già sulle scale mi dice: «Se verrà un'altra 
volta, potremo parlare più a lungo». Io corro giù, non prendo l'ascensore. Non lo vedrò mai più̀ ${ }^{80}$. giudiziaria che vedeva coinvolti i figli di Bassani e la sua compagna americana intorno alla sua eredità. Per il suo ottantesimo compleanno nel 1996, e in occasione della sua scomparsa nel 2000, uscirono, come di consueto, una serie di articoli bio-bibliografici a carattere commemorativo, e quando nel $2004 \mathrm{fu}$ pubblicata una nuova traduzione del Gattopard ${ }^{81}$ - basata sulla nuova edizione italiana, realizzata a partire dal manoscritto di Lampedusa, e non dal dattiloscritto utilizzato da Bassani - si scrisse ancora una volta del ruolo che aveva avuto lo scrittore ferrarese nella prima edizione dell'opera. Neppure il centenario della nascita, nel 2016, ha cambiato molto le cose.

\section{«Affinità elettive»}

È possibile trovare tracce di una ricezione 'creativa' dell'opera di Bassani nella letteratura tedesca? Abbiamo già visto che l'impiego poetico della memoria in Bassani ha potuto influire su Alfred Andersch, anche se la cosa resta per ora da dimostrare. Tra i numerosi autori di lingua tedesca che hanno collaborato a «Botteghe oscure» è difficile reperire qualcuno che sia stato in stretto contatto con Bassani o che, addirittura, si sia occupato della sua opera. Un'eccezione di rilievo è costituita da Hilde Domin, di cui tre poesie - Landschaft bei Cádiz [Paesaggio vicino a Cadice], Jenseits des Bergs [Al di là della montagna] e Osterwind [Vento di Pasqua] - sono state pubblicate sul numero XXV di «Botteghe oscure». Nel Deutsches Literaturarchiv a Marbach è conservata una lettera in italiano di Domin a Bassani, datata 5 giugno 1966, in cui, riferendosi a Cesare Cases, loro conoscenza comune, la scrittrice chiedeva se anche Bassani si sarebbe recato a New York al congresso internazionale del Pen, e se lo avesse potuto incontrare in quell'occasione. Domin accludeva, inoltre, il testo di una conferenza, scritta probabilmente in inglese, che nella sua lettera intitolava «La funzione della poesia nella società moderna». Si trattava probabilmente del testo di una conferenza che aveva tenuto nel 1964 in diverse università americane e che aveva pubblicato anche in versione tedesca presso il comune editore Piper di Monaco nel 1968, nel suo libro Wozu Lyrik heute. Dichtung und Leser in der gesteuerten Gesellschaft [Perché la lirica oggi? Poesia e lettori in una società manovrata] con il titolo Wozu Lyrik heute? Lyrik und Gesellschaft [Perché la lirica oggi? Lirica e società].

Ancor più importante, però, è una poesia allegata alla lettera. Domin la definisce «un poema che è un manifesto politico». Era stata pubblicata su «Die Zeit» il 20 maggio 1966. A Bassani la presenta come un appello contro un giornale neonazista, che solo lei avrebbe potuto scrivere. Era infatti l'unica poetessa a essere tornata in Germania dopo l'esilio, e incontrava ora molte difficoltà di ogni genere. Lo informava anche del fatto che aveva trovato un traduttore italiano per le sue poesie e che i primi esiti le parevano soddisfacenti. La poesia che gli mandava era un testo che nell'edizione di tutte le sue poesie, pubblicata nel 1987, porta il titolo Graue Zeiten [Tempi grigi] ${ }^{82}$, ma che nella redazione del 1966, destinata alla pubblicazione sul quotidiano, recava il titolo Deutsche National-Zeitung und Soldatenzeitung. Auflage etwa 94.000 [Quotidiano nazionale tedesco e Quotidiano dei soldati. Tiratura di circa 94000 copie]. Un'altra ristampa della poesia, in un volume curato dalla stessa autrice, includeva la seguente nota esplicativa: «Scritta nella primavera del 1966 sotto l'impressione dell'improvvisa comparsa in tutte le edicole dei 
caratteri cubitali della "National-Zeitung" e delle statistiche allarmanti [...] sull'aumento del radicalismo di destra nella Repubblica Federale Tedesca» ${ }^{83}$. È evidente che nel 1966 la Domin vedeva Bassani e Cesare Cases come istanze morali che dovevano essere informate di una reviviscenza dell'antisemitismo e del nazionalsocialismo in Germania.

Nella corrispondenza di Domin con Piper-Verlag conservata a Marbach si trova una sua traduzione della poesia di Bassani Le leggi razziali, che la poetessa aveva realizzato, non prima del 1982, basandosi sull'edizione delle poesie di Bassani pubblicata in quell'anno da Mondadori con il titolo In rima e senza. Finora non siamo riusciti a verificare se questa traduzione sia mai stata pubblicata. Non figura nell'edizione curata da Domin delle sue traduzioni poetiche, e la traduzione tedesca de Le leggi razziali, pubblicata da Piper-Verlag nell'edizione bilingue delle poesie di Bassani e realizzata da Michael Marschall von Bieberstein, è completamente diversa. La traduzione della Domin comincia così:

Die Magnolie die genau in der Mitte

des Gartens unseres Hauses in Ferrara steht, sie ist's die wiederkehrt

in fast allen meinen Büchern ${ }^{84}$.

La Domin, che nel 1932 si era trasferita in Italia con il marito per motivi di studio, non rientrò più in Germania dopo il 1933, e sarà in seguito costretta a lasciare anche l'Italia, nel 1938, per via delle leggi razziali. A posteriori, l'autrice ebbe modo di riflettere sul fatto che la sua permanenza in Italia non aveva influito sulla sua scrittura. In occasione della consegna del premio Rainer Maria Rilke per la lirica nel 1976, si rammentò dell'effetto che la lettura di Rilke aveva avuto su di lei quando era ancora in esilio, mentre la letteratura italiana non aveva lasciato alcuna traccia nella sua produzione letteraria:

È strano che la letteratura italiana non abbia mai interferito, durante i sette anni del nostro soggiorno italiano, con l'amore per quella tedesca, e nemmeno la letteratura inglese, quando nel 1939 lasciammo l'Italia ed emigrammo in Inghilterra. Le cose andarono diversamente con la letteratura spagnola ${ }^{85}$.

Ciò che può essere vero per il periodo dell'esilio italiano, non vale più per il periodo successivo al rientro in Germania all'inizio degli anni 1960. Almeno Bassani divenne per Domin un importante punto di riferimento in Italia, se non altro per quanto riguarda le reazioni letterarie all'antisemitismo tedesco o italiano. Non è pertanto possibile concordare appieno con Nadia Centorbi quando scrive che per Domin, dal punto di vista retrospettivo della metà degli anni '80, quando poté tornare a Roma con una borsa di studio di Villa Massimo, l'Italia era stata «quella seconda patria destinata a imprimersi nella memoria della poetessa nella sua ideale trasformazione, mai scalfita dall'esperienza del fascismo e dall'applicazione delle leggi razziali» ${ }^{86}$. Se Domin ha tradotto in tedesco, dopo il 1982, la poesia di Bassani sulle leggi razziali, è lecito supporre che tanto quella legislazione quanto la conseguente persecuzione degli Ebrei in Italia, che la poetessa aveva sperimentato in prima persona, dovevano essere molto presenti alla sua mente, e che Bassani fungeva da catalizzatore dei suoi ricordi.

Oltre che in Andersch e nella Domin, una ricezione 'produttiva' di Bassani in lingua tedesca si riscontra principalmente in W. G. Sebald. Di recente Ben Hutchinson ha potuto dimostrare che Sebald si era occupato intensamente delle opere di Bassani, avendo rinvenuto chiare tracce di lettura nelle copie dei testi di Bassani di proprietà dello scrittore conservati nel Deutsches Literaturarchiv di Marbach ${ }^{87}$. Come già per 
Andersch, così anche per Sebald pare opportuno parlare di 'affinità elettive' più che di ricezione diretta. Hutchinson prova in modo convincente che la lettura sebaldiana delle traduzioni tedesche di Bassani, e in particolare del volume curato da Eberhard Schmidt, dovette aver luogo alla fine del $1998^{88}$. Si può tuttavia supporre che Sebald avesse letto già prima Il Giardino dei Finzi-Contini, dato che il campo da tennis abbandonato in un parco inselvatichito, dove il dott. Henry Selwyn accoglie il narratore nel racconto che dà il titolo alla raccolta Die Ausgewanderten [Gli emigrati], ricorda da vicino quello dei Finzi-Contini.

Un seguito al romanzo di Bassani, incentrato sulla protagonista femminile, è stato proposto da Waltraud Mittich nel racconto Micòl, uscito nel 2016, l'anno del centenario della nascita di Bassani ${ }^{89}$. L'idea di fondo, secondo cui Micòl sarebbe riuscita a salvarsi dal campo di concentramento e avrebbe condotto, nel periodo postbellico, una vita palpitante di donna emancipata in giro per il mondo, è talmente imbarazzante che risparmio al lettore le citazioni. In fondo non si tratta tanto di un omaggio a Bassani quanto di una lettura di autoimmedesimazione, dato che alla «narratrice», come è ripetuto più volte, interessa sempre «e soprattutto» la narratrice stessa, e Micòl le serve sopprattutto da schermo su cui proiettare una vita di donna emancipata, descritta con un tocco di kitsch.

A cent'anni dalla nascita e a diciassette dalla morte di Bassani si può dire, in conclusione, che nei paesi di lingua tedesca non c'è stata una ricezione veramente 'produttiva' della sua opera, e anche quei pochi autori 'noti' che si sono confrontati con lo scrittore ferrarese, lo hanno fatto, più che altro, per una sorta di 'affinità elettiva', perché s'interessavano a temi analoghi come la rappresentabilità in letteratura dei processi della memoria, e più raramente per una ricezione o appropriazione diretta - con l'unica eccezione della traduzione di Le leggi razziali di Hilde Domin. Ma se prendiamo come parametro le continue riedizioni delle sue opere, se non altro le più note, come Gli occhiali d'oro e Il Giardino dei Finzi-Contini, ci sembra di poter affermare che il pubblico di lingua tedesca ha dimostrato un interesse duraturo per le opere di Bassani. In occasione del ritrovamento del manoscritto originale del Giardino, che faceva parte del lascito di Teresa Foscari Foscolo, nell'aprile del 2016, Dirk Schümer ha indicato su «Die Welt» ciò che in Bassani ha chiaramente affascinato lettori e lettrici diversi fra loro come Andersch, Domin o Sebald e che continua ad affascinare i lettori di oggi: «Il segreto della grande letteratura non sta nell'immediatamente visibile, ma nel non-detto» ${ }^{90}$.

\section{NOTE}

1. L'articolo è stato tradotto dal tedesco da Roberta Martignon con la collaborazione di Francesco Bonelli, Giulia Cacciatore ed Enzo Neppi.

2. P. Prebys, Giorgio Bassani, vol. II: La memoria critica su Giorgio Bassani, [Roma], Portia Prebys, 2010.

3. Sto, Giorgio Bassani, Die Gärten der Finzi-Contini, «Landeskriminalblatt Niedersachsen», 4 giugno 1967, in P. Prebys, Giorgio Bassani, cit., p. 106 [nº 1284 della bibliografia]. 
4. Anon., Bücher von Kennern, «Norddeutsche Schlachter-Zeitung», 20 gennaio 1968 [nº 1344 della bibliografia].

5. G. Manganelli, Unschluss, trad. ted. di I. Schnebel-Kaschnitz, Berlin, Wagenbach, 1978.

6. Durante la sua residenza a Roma, presso Villa Massimo (1982-1985), Krüger conobbe personalmente Bassani.

7. M. Krüger, Der Regenstaat. Giorgio Manganellis satirischer Traktat „Unschluß“, «Die Zeit», 15 settembre 1978 (qui e nel seguito dell'articolo, sono nostre le traduzioni dal tedesco).

8. Un medico di Ferrara, solo nella seconda edizione del 1985 il titolo divenne più correttamente Die Brille mit dem Goldrand.

9. G. Bassani, Die Gärten der Finzi-Contini, trad. ted. di H. Schlüter, München, Piper, 1963;

G. Bassani, Ferrareser Geschichten, trad. ted. di H. Schlüter, München, Piper, 1964.

10. G. Bassani e M. Soldati, Venedig, Stadt auf 118 Inseln [Venezia, città dalle 188 isole], fotografie di

G. Berengo-Gardin, trad. ted. di M. e J. Keller, Starnberg, Keller, 1965.

11. G. Bassani, Die Gärten der Finzi-Contini, trad. ted. di Herbert Schlüter, Zürich, Buchklub Ex libris, 1965, e ivi, Stuttgart, Hamburg, Deutscher Bücherbund, 1965.

12. G. Bassani, Hinter der Tür, trad. ted. di Herbert Schlüter, München, Piper, 1967.

13. G. Bassani, Die Gärten der Finzi-Contini, trad ted. di Herbert Schlüter, Frankfurt am Main, Hamburg, Fischer, 1967.

14. Sulla dibattuta questione se Andersch, nel 1944, disertò in Italia dalla Wehrmacht, come racconta nel suo testo Die Kirschen der Freiheit. Ein Bericht, o se fu catturato da soldati americani, cfr. J. Döring, F. Römer e R. Seubert, Alfred Andersch desertiert. Fahnenflucht und Literatur (19441952), Berlin, Verbrecher Verlag, 2015.

15. Cfr. I. M. Battafarano, Vom Sinn des Erzählens. Andersch über Bassani, in M. Korolnik e A. Korolnik-Andersch (a cura di), Sansibar ist überall. Alfred Andersch. Seine Welt - in Texten, Bildern, Dokumenten, München, edition text+kritik, 2008, pp. 128-141; D. Scuto, Das römische Paradies, in Sansibar ist überall, cit., pp. 142-149.

16. Cfr. I. M. Battafarano, Vom Sinn des Erzählens, cit., pp. 139-140, e S. Reinhardt, Alfred Andersch. Eine Biographie, Zürich, Diogenes, 1990, p. 369.

17. Nel 1975 Volk und Welt pubblicò una seconda ristampa delle Ferrareser Geschichten.

18. G. Bassani, Der Geruch von Heu, trad. ted. di H. Schlüter, München, Zürich, Piper, 1974.

19. La vendita delle opere crebbe però continuamente, anche se non in maniera esponenziale. I Finzi-Contini uscirono nel 1975 per Piper in una quarta ristampa in circa 12000 esemplari.

20. G. Bassani, Die Gärten der Finzi-Contini, trad. ted. di H. Schlüter, con una postfazione di J. Meinert, Berlin, Volk und Welt, 1981.

21. E. Kanduth, Giorgio Bassanis Il giardino dei Finzi-Contini im Spiegel der Varianten, «Italienische Studien», nº 6, 1983, pp. 105-123.

22. G. Bassani, Die Gärten der Finzi-Contini, nuova ed., München, Zürich, Piper, 1984; G. Bassani, Ferrareser Geschichten, nuova edizione, München, Piper, 1985.

23. G. Bassani, In einem alten italienischen Garten. Gedichte, italienisch/deutsch [In un vecchio giardino italiano. Poesie, in edizione bilingue], scelte e tradotte da M. Marschall von Bieberstein, con una prefazione di H. W. Wittschier, München, Zürich, Piper, 1991.

24. G. Bassani, Erinnerungen des Herzens [Ricordi del cuore], con testi di G. Cattaneo, P. Ravenna ed E. Schmidt, a cura di E. Schmidt, München, Zürich, Piper, 1991.

25. Piper ha continuato a pubblicare i Finzi-Contini sotto la nuova direzione (1995, 1996, 1997, 2002, 2004). Edizioni dell'opera sono uscite anche presso gli editori Wagenbach (2000) e Manesse (2005).

26. Sull'opera di Bassani all'interno del programma editoriale di Piper Verlag, vedi E. Ziegler, 100 Jahre Piper. Die Geschichte eines Verlags, München, Zürich, 2004, pp. 255-264. 
27. Nel 2005 Die Gärten der Finzi-Contini, sempre nella stessa traduzione, uscì anche a Zurigo: G. Bassani, Die Gärten der Finzi-Contini, trad. ted. di H. Schlüter, postfazione di U. Stempel, Zürich, Manesse, 2005.

28. G. Bassani, Der Reiher, trad. ted di H. Schlüter, Berlin, Wagenbach, 2007.

29. G. Bassani, Hinter der Tür, trad. ted. di H. Schlüter, Berlin, Wagenbach, 2008.

30. G. Bassani, Die Passeggiata, in C.W. Macke (a cura di), Bologna und Emilia Romagna. Eine literarische Einladung [Un invito letterario], Berlin, Wagenbach, 2009, pp. 84-88 (si tratta di un brano de La passeggiata prima di cena).

31. Wagenbach non dà informazioni sul numero di copie stampate, ma Piper, con la quattordicesima ristampa dei Finzi-Contini (1995) raggiunse le 93000 copie, e si può quindi ipotizzare che in totale, dal 1963 ad oggi, siano state vendute più di 100000 copie del romanzo. Se si calcolano anche le copie vendute nella DDR, le cui case editrici stampavano per un pubblico di massa, è possibile stimare realisticamente un totale di 200000 copie.

32. Da ricordare qui una serie di tesi universitarie su Bassani: R. Mader, „È bello raccontare i guai passati“? Konstanten einer "Literatur der Gezeichneten“ im Kontext des italienischen Faschismus am Beispiel dreier jüdischer Zeitzeugen: Primo Levi, Giorgio Bassani, Natalia Ginzburg, Diss. Phil., Freiburg im Breisgau, 1999; G. Waste, Jüdische Identität im Zeichen von Bildlichkeit und Erinnerung in Giorgio Bassanis Romanzo di Ferrara, Würzburg, Königshausen und Neumann, 2003; D. Gomille, Den Holocaust erzählen. Untersuchungen zum erinnernden Schreiben in Giorgio Bassanis Romanzo di Ferrara, Diss. Phil., Freiburg im Breisgau, 2011; I. von Treskow, Judenverfolgung in Italien (1938-1945) in Romanen von Marta Ottolenghi Minerbi, Giorgio Bassani, Francesco Burdin und Elsa Morante, Wiesbaden, Harrassowitz, 2013.

33. Cfr. H. C. Kennedy, The Ideal Gay Man. The Story of "Der Kreis", New York, Haworth Press, 1999; una versione digitalizzata della rivista è accessibile sul sito della biblioteca universitaria dell'ETH Zürich, cfr. <www.e-periodica.ch/digbib/volumes?UID=kre-003>.

34. C. Helder, Giorgio Bassani: Ein Arzt aus Ferrara, «Der Kreis. Le Cercle. The Circle», vol. 28, $\mathrm{n}^{\circ} 12$, 1960, pp. 7-8.

35. Ivi, p. 8.

36. Ibid.

37. F. Tumler, Vereinsamung, «Zeitwende. Die neue Furche», vol. 32, 1961, pp. 558-559.

38. Ibid.

39. G. Tomasi di Lampedusa, Der Leopard, trad. ted. di C. Birnbaum, München, Piper, 1959.

40. G. Tomasi di Lampedusa, Die Sirene und andere Erzählungen [Le sirene e altri racconti], trad. ted. di C. Birnbaum, postfazione di G. Bassani, München, Piper, 1961.

41. Cfr. Botteghe oscure. Index 1949-1960, compiled by several hands, with an introduction by Archibald MacLeish, including an index to "Commerce" (1924-1932), Middletown (Connecticut), Wesleyan University Press, 1964, pp. 74-79.

42. Di Bachmann uscirono sul quaderno XIV (1954) Lieder von einer Insel e Nebelland, nel quaderno XIX (1957) Hôtel de la Paix, Nach dieser Sintflut, Liebe: Dunkler Erdteil, Exil e Mirjam; di Celan nel 1956 nel quaderno XVII Vor einer Kerze. Per il numero comune del 1958 (quaderno XXI), Nelly Sachs scrisse otto poesie e portò sei poesie di Walter Höllerer, una di Günter Grass, sei di Hans Magnus Enzensberger e una traduzione di Celan del Bateau ivre di Rimbaud. Nella corrispondenza degli autori non si tratta di Bassani quanto dell'editrice Marguerite Caetani, cfr. Herzzeit. Ingeborg Bachmann-Paul Celan. Der Briefwechsel, con la corrispondenza fra P. Celan e M. Frisch, fra I. Bachmann e G.Celan-Lestrange, a cura di B. Badiou, H. Höller, A. Stoll e B. Wiedemann, Frankfurt am Main, Suhrkamp, 2008, lettere del 9 novembre 1957, del 14 novembre 1957 e del 16 novembre 1957, nonché le note alle pp. 281-282.

43. M. von Zitzewitz, Ein Krampf um Rom [Un crampo per Roma], «Die Zeit», 24 agosto 1962. Il titolo dell'articolo è un rimando parodico al romanzo di Felix Dahn, Ein Kampf um Rom [Una battaglia per Roma] (1876), che racconta la caduta degli Ostrogoti nella tarda antichità, e che era ancora noto 
intorno al 1960, come mostra anche l'omonimo film con Orson Welles (regia di Robert Siodmak, 1968).

44. Anon., Giorgio Bassani: Die Gärten der Finzi-Contini, «Der Spiegel», 25 dicembre 1963, p. 105.

45. Die Stimme des Buchhandels, «Die Zeit», 11 ottobre 1963.

46. Ibid.

47. A. Silenius, Die Erinnerung der „verlorenen Zeit“, «Tribüne. Zeitschrift zum Verständnis des Judentums», vol. II, $\mathrm{n}^{\circ}$ 8, 1963, pp. 871-874.

48. W. Grözinger, Die Gefahr der großen Stoffe, «Hochland», vol. LVI, n² 2, dicembre 1963, pp. 169-178, cit. da W. Grözinger, Panorama des internationalen Gegenwartsromans. Gesammelte „Hochland“-Kritiken 1952-1965, ed. a cura di E. Rotermund e H.Ehrke-Rotermund, Paderborn, Schöningh, 2004, p. 411.

49. Ivi, p. 412.

50. Anon., Giorgio Bassani: Ferrareser Geschichten, «Der Spiegel», 16 dicembre 1964.

51. Ibid.

52. G. Bassani, Hinter der Tür, aus dem Italienischen von H. Schlüter, München, Piper, 1967.

53. G. Bassani, Die Gärten der Finzi-Contini, aus dem Italienischen von H. Schlüter, Frankfurt am Main, Hamburg, Fischer, 1967.

54. I. M. Battafarano, Vom Sinn des Erzählens, cit., pp. 139-140.

55. Ivi, p. 129.

56. Ivi, p. 137.

57. Kulturpreis der Stadt Dortmund, Nelly-Sachs-Preis 1969, Giorgio Bassani oder vom Sinn des Erzählens, Laudatio Alfred Andersch, Kulturamt der Stadt Dortmund, 1969, p. 3

58. A. Andersch, Auf den Spuren der Finzi-Contini [Sulle tracce dei Finzi-Contini], «Merkur», vol. XXI, $\mathrm{n}^{\circ}$ 10, 1967, pp.943-955, ora in Id., Essayistische Schriften 3, Gesammelte Werke in zehn Bänden, edizione commentata a cura di D. Lamping, vol. 10, Zürich, Diogenes, 2004, pp. 71-89.

59. Nelly Sachs a Alfred Andersch, Stoccolma, 7 maggio 1968, in R. Dinesen e H. Müssener (a cura di), Briefe der Nelly Sachs, Frankfurt am Main, Suhrkamp, 1985, p. 317.

60. G. Bassani, Dank [Ringraziamento], in Giorgio Bassani. Ansprachen und Dokumente zur Verleihung des Kulturpreises der Stadt Dortmund Nelly-Sachs-Preis am 7. Dezember 1969, Dortmund, Mitteilungen aus dem Literaturarchiv, 1971, p. 31.

61. Ibid.

62. Ivi, p. 29: «Non conoscendo abbastanza bene il tedesco, non ho capito tutto ciò che Lei ha appena detto. Mi è parso tuttavia che il suo discorso di oggi non si sia allontanato di molto da ciò che Lei ha scritto nel saggio pubblicato in Merkur nel 1967 e in Écriture quest'anno».

63. V. Kammandel, Die produktive Rezeption zeitgenössischer italienischer Erzähler in der westdeutschen Nachkriegsliteratur. Studien zum Werk von Alfred Andersch und Hans Erich Nossack, Heidelberg, Winter, 2012, pp. 152-153.

64. Kulturpreis der Stadt Dortmund, Nelly-Sachs-Preis 1969, Giorgio Bassani oder vom Sinn des Erzählens, cit., p. 11.

65. J. Améry, Lettera a A. Andersch, Bruxelles, 19 luglio 1972, in J. Améry, Werke, vol. 8: Ausgewählte Briefe 1945-1978, a cura di Gerhard Scheit, Stuttgart, Klett-Cotta, 2007, p. 401.

66. T. Kienlechner, Ausflug zum Tod. Giorgio Bassanis «Der Reiher», «Die Zeit», 1 maggio 1970.

67. J. Meinert, Nachwort, in G. Bassani, Die Gärten der Finzi-Contini, Berlin, Verlag Volk und Welt, 1970, p. 325.

68. Ivi, p. 326-327.

69. Ivi, p. 328.

70. Meinert afferma che questa intervista era stata rilasciata alla rivista Il Ponte nel 1960 ma per ora non siamo riusciti a trovarne traccia, né sul Ponte né in altra sede.

71. J. Meinert, Nachwort, cit., p. 328. 
72. Vedi E. Schmidt, Auf den Spuren der Finzi-Contini. Ein Gang durch Giorgio Bassanis Ferrara - heute, in G. Bassani, Erinnerungen des Herzens, cit., p. 134, che a sua volta cita le parole di Bassani, ma senza indicarne la fonte. I segni lasciati da Sebald nella sua copia di questo volume sono state esaminate da B. Hutchinson in Erzähltechnik II: „Der Erzähler als Schutzengel“. Sebalds Lektüre von Giorgio Bassani, in B. Hutchinson, W. G. Sebald. Die dialektische Imagination, Berlin, De Gruyter, 2009, pp. 57-76. Il passo citato si trova a p. 61.

73. J. Meinert, Nachwort, cit., p. 335.

74. Ivi, p. 342.

75. R. Sanders, Das kleine Universum Ferrara, «Die Zeit», 21 novembre 1974.

76. R. Sanders, Das kleine Universum Ferrara, cit.

77. G. Ammann, Ein Wahlverwandter Marcel Prousts, Mutmassungen über das lange Schweigen von Giorgio Bassani [Affinità elettive con Marcel Proust. Congetture sul lungo silenzio di Giorgio Bassani], «Orte. Schweizer Literaturzeitschrift», $n^{\circ}$ XXXI, 1980, pp. 13-17 (il passo citato è a p. 13).

78. Ivi, p. 17.

79. G. Seibt, Der Kuß. Ein Abend bei dem Schriftsteller Giorgio Bassani, «Frankfurter Allgemeine Zeitung», 2 febbraio 1995, p. 29.

80. Ibid.

81. G. Tomasi di Lampedusa, Der Gattopardo: Roman, trad. tedesca di G. Waeckerlin Induni, München, Piper, 2004.

82. H. Domin, Gesammelte Gedichte, Frankfurt am Main, Fischer, 1987, pp. 340-344.

83. H. Domin (a cura di), Nachkrieg und Unfrieden. Gedichte als Index 1945-1970 [Periodo postbellico e discordia. Poesie come indice 1945-1970], Neuwied, Luchterhand, 1970, p. 93.

84. H. Domin, Die Rassengesetze, Deutsches Literaturarchiv Marbach, Domin, Hilde, R. Piper du Co., München, 1980-1989, HS.2007.0002. Così invece l'incipit della traduzione di Michael Marschall von Bieberstein: «Die Magnolie wächst genau in der Mitte des Gartens / unseres Hauses in Ferrara und eben sie ist es auch / die in fast allen meinen Büchern / wiederkehrt» (G. Bassani, Erinnerungen des Herzens, cit., p. 53).

85. H. Domin, Ins Exil mit Goethe, Heine, Rilke, Joyce. Dankrede bei der Entgegennahme des Rainer Maria Rilke-Preises für Lyrik 1976, in H. Domin, Aber die Hoffnung. Autobiographisches aus und über Deutschland, Frankfurt am Main, Fischer, 1993 (Piper, 1982), pp.144-148, cit. a p. 145. Trad. italiana in N. Centorbi, «Mit der Antike, mit der Renaissance und dem Barock, aber nicht mit Mussolini und der Gegenwart». Riflessioni dall'esilio italiano di Hilde Domin, in A. Schininà e M. Bonifazio (a cura di), Un luogo per spiriti più liberi. Italia, Italiani ed esiliati tedeschi, Roma, Artemide, 2014, pp. 65-92, cit. a p. 90.

86. N. Centorbi, ivi, p. 92.

87. B. Hutchinson, Erzähltechnik II, cit.

88. Ivi, pp. 58-59.

89. W. Mittich, Micòl, Innsbruck, Laurin, 2016.

90. D. Schümer, Das letzte Geheimnis der Finzi-Contini, «Die Welt», 27 aprile 2016.

\section{RIASSUNTI}

Nei paesi di lingua tedesca, Giorgio Bassani è uno degli autori italiani più letti del XX secolo. Benché la sua opera sia stata ampiamente recepita e commentata, e sia ancora in gran parte 
disponibile sul mercato librario, sono pochi gli scrittori di lingua tedesca (Andersch, Domin, Sebald), che si confrontano con i testi di Bassani in modo 'produttivo'. Questo saggio cerca di ricostruire sia le diverse fasi della ricezione critica di Bassani, sia le tracce di un confronto artistico e creativo con la sua opera.

Giorgio Bassani zählt im deutschsprachigen Raum zu den am meisten gelesenen italienischen Autoren des 20. Jahrhunderts. Obwohl sein Werk in der Öffentlichkeit breit rezipiert und kommentiert wurde und bis heute fast vollständig im Buchhandel verfügbar ist, finden sich nur wenige deutschsprachige Autoren (Andersch, Domin, Sebald), die sich produktiv auf Bassanis Texte beziehen. Der Essay versucht, sowohl die Phasen der Rezeption als auch die Spuren produktiven Umgangs mit Bassanis Werk nachzuzeichnen.

Dans les pays d'expression allemande, Giorgio Bassani est l'un des auteurs italiens les plus lus du $\mathrm{xx}^{\mathrm{e}}$ siècle. Son œuvre a été largement accueille et commentée dans la presse et dans les milieux culturels et elle est encore très présente sur le marché libraire. Néanmoins, peu d'écrivains d'expression allemande (Andersch, Domin, Sebald) se sont rapportés de manière productive aux textes de Bassani. Cet essai tente de reconstruire aussi bien les différentes phases de la réception critique de Bassani, que les traces d'un dialogue poétique avec son œuvre.

\section{INDICE}

Parole chiave : ricezione tedesca, memoria, traduzione, leggi razziali, nazionalsocialismo, Nelly Sachs, Hilde Domin, esilio, Alfred Andersch, W. G. Sebald, Jean Améry, Marcel Proust

Schlüsselwörter : deutschsprachige Rezeption, Erinnerung, Rassengesetze, Nationalsozialismus, Nelly Sachs, Hilde Domin, Exil, Alfred Andersch, W. G. Sebald, Jean Améry, Marcel Proust

Mots-clés : réception allemande, mémoire, traduction, lois raciales, Nelly Sachs, Hilde Domin, Alfred Andersch, W. G. Sebald, Jean Améry

\section{AUTORE}

\section{OLAF MÜLLER}

Philipps Universität Marburg 